\title{
Dual Band CPW-Fed Double Monopole Antenna for 2.4/5.8 GHz ISM band Medical Applications
}

\author{
Chaïmaâ Kissi \\ Electronics and Telecommunication \\ Systems Research Group, National \\ School of Applied Sciences (ENSA) \\ Ibn Tofail University \\ Kenitra, Morocco \\ chaimaakissi1@gmail.com
}

Sami Myllymäki

Microelectronics Research Unit,

Faculty of Information Technology and

Electrical Engineering

University of Oulu

Oulu, Finland

sami.myllymaki@oulu.fi

\author{
Mariella Särestöniemi \\ Centre for Wireless Communications, \\ Faculty of Information Technology and \\ Electrical Engineering \\ University of Oulu \\ Oulu, Finland \\ mariella.sarestoniemi@oulu.fi
}

Mohamed Nabil Srifi

Electronics and Telecommunication

Systems Research Group, National

School of Applied Sciences (ENSA)

Ibn Tofail University

Kenitra, Morocco

srifimn@gmail.com

Carlos Pomalaza-Raez

Department of Electrical and Computer

Engineering

Purdue University

Indiana, USA

cpomalaz@purdue.edu

\author{
Marko Sonkki \\ Centre for Wireless Communications, \\ Faculty of Information Technology and \\ Electrical Engineering \\ University of Oulu \\ Oulu, Finland \\ marko.sonkki@oulu.fi
}

Heli Jantunen

Microelectronics Research Unit,

Faculty of Information Technology and

Electrical Engineering

University of Oulu

Oulu, Finland

heli.jantunen@oulu.fi

\begin{abstract}
The conception and realization of a dual-band coplanar waveguide fed double monopole antenna, working at 2.4 and $5.8 \mathrm{GHz}$ Industrial, Scientific and Medical (ISM) bands, is presented in this paper. To operate effectively at the aforementioned bands, the designed antenna consists of a symmetrical folded long monopole and a short monopole which are responsible of the resonant frequencies at 2.45 and $5.8 \mathrm{GHz}$, respectively. Furthermore, both monopoles are fed by a coplanar waveguide. The symmetrical configuration allows the proposed antenna to obtain symmetrical radiation patterns. Hence, an omnidirectional radiation pattern is obtained at 2.45 and $5.8 \mathrm{GHz}$. A detailed parametric study and antenna parameters are reported and the proposed antenna is designed, fabricated and measured in free space. The proposed antenna has a compact size with the overall dimensions of $30 \mathrm{~mm} \times 27 \mathrm{~mm} \times 1.6 \mathrm{~mm}$. It is concluded from the measured results, that the presented antenna could be considered for applications operating at 2.4 and $5.8 \mathrm{GHz}$ ISM bands. Next, a metallic reflector was introduced to the planar structure in order to improve the gain and provide better directivity for onbody applications, for instance wireless capsule endoscopy systems. For this specific application, the antenna will act as receiving antenna and hence will be held by the patient, which requires a primary on/in-body investigation next to the small intestine region (complex organ of the gastrointestinal tract), as discussed in the paper using multi-layer and voxel models. It is concluded that the proposed antenna structure is a good receiving antenna for wireless capsule endoscopy systems.
\end{abstract}

Keywords-coplanar waveguide (CPW), dual-band, folded long monopole antenna, multi-layer model, omnidirectional pattern, small intestine, short monopole antenna, voxel model

\section{INTRODUCTION}

The use of wireless and mobile communication systems has been increased exponentially last decades. The scope of the dual-band antennas has been extended to a wide range of applications, especially using $2.4 / 2.45 \mathrm{GHz}$ and $5.8 \mathrm{GHz}$
Industrial, Scientific and Medical (ISM) bands. At this frequency range, an amount of dual-band antennas is available in the literature, serving diverse applications such as: Wireless Body Area Networks (WBAN) [1-4], RFID [5], WLAN [6], biomedical purposes [7], and wireless communication devices [8]. In this perspective, various kinds of dual-band antennas have been designed to meet the requirements of the listed applications. However, the common requirements in the conception and realization antenna process remain the compact size, suitable radiation characteristics, simple and flexible structures, low profile, reasonable gain and high efficiency adequate to the aimed application. Nowadays, the challenging and the intensively investigated medical application is wireless capsule endoscopy. Several frequencies are used for this application but a wide range of researches has been conducting investigations at $2.4 / 2.45 \mathrm{GHz}$ ISM band. By staying restricted to this frequency medical band, several papers presenting capsule antennas designed to operate at this 2.4$2.48 \mathrm{GHz}$ frequency range can be found in literature [9][10][11]. In [10], an in-vivo test environment is proposed to assess the capsule antenna performances by directly measuring the antenna features in rats without evaluating the communication link with an external antenna. However, to evaluate the communication link of the capsule endoscope antenna, an external antenna is highly needed and for this end few papers were published to address this communication issue. For example, a proposed capsule antenna operating at $2.4 \mathrm{GHz}$ ISM band is proposed in [12] and the communication link features were evaluated by using an external dipole receiving antenna with a size of $60 \mathrm{~mm}$-length and $60 \mathrm{~mm}$-width. In this reference, the focus was giving on the capsule antenna and letting aside the details of the receiving antenna (lack of its gain at $2.4 \mathrm{GHz}$ ). 
The only available information regarding the receiving antenna is the $-10 \mathrm{~dB}$ bandwidth of $2.20-2.83 \mathrm{GHz}$. In [12], an omnidirectional receiving antenna is used for the budget and communication link evaluation. The external antenna has a realized gain of $1 \mathrm{~dB}$, operating at $2.41-2.47 \mathrm{GHz}$ and is made of two elements: a top-loaded monopole and a dipole of $15 \mathrm{~mm}$ and $30 \mathrm{~mm}$ radiuses, respectively. In [13], a horn antenna is used to excite the capsule in the measurement achieving a gain of $7.9 \mathrm{~dB}$, however the antenna limitation is the huge size unsuitable to realistic and practical operation. According to the related work cited previously and up to the author's knowledge the receiving antennas used in published works are only designed for general communications and does not target the signal ability to travel and propagate through the human body which should be highly considered in the investigations beforehand. For this aim, this paper comes to provide a directive wearable antenna operating at $2.4 / 5.8 \mathrm{GHz}$ ISM band for in-body communications. The proposed antenna is targeted to radiate normally towards the human body and be able to reach the small intestine tract and hence establish a reliable and efficient communication link between the wearable antenna and the swallowed capsule antenna (travelling the small intestinal tract). The author's intention is to compare the antenna behavior in terms of matching, directivity and power consumption estimation by the human body at lower and higher ISM band frequencies $2.4 \mathrm{GHz}$ and $5.8 \mathrm{GHz}$, respectively which has not been addressed in literature for wireless capsule endoscopy research topic. This way, in coming paper, a capsule antenna operating at 2.4/5.8 GHz ISM band will be designed properly and then the communication and budget link will be evaluated using the proposed external antenna by simulations and confirmed by measurements.

In this paper, a novel dual-band planar antenna covering 2.4/5.8 GHz ISM bands is proposed. The antenna consists of a folded long monopole antenna and a short monopole antenna. By feeding the two monopoles with a CPW (coplanar waveguide), omnidirectional radiation patterns are obtained at 2.45 and $5.8 \mathrm{GHz}$. This antenna structure is completed by reflector to achieve better directivity and good gain of $6.83 \mathrm{~dB}$ and $3.39 \mathrm{~dB}$ at $2.4 \mathrm{GHz}$ and $5.8 \mathrm{GHz}$, respectively. Then the final antenna structure is assessed next to multi-layer and voxel models which confirm the suitability of the antenna for Body Area Networks applications, in particular wireless capsule endoscopy localization systems.

\section{Planar AntenNa STRUCTURE}

\section{A. Antenna configuration and simulation analysis}

The overall conception of the proposed antenna is depicted in Fig. 1. The radiating element and the ground plane are both printed on the front side of FR-4 substrate material (relative permittivity of 4.3 and tangent loss of 0.025 ) with a length of $30 \mathrm{~mm}$, width of $27.04 \mathrm{~mm}$, and height of $1.6 \mathrm{~mm}$. The proposed antenna consists of two radiating elements, symmetrical folded monopole and a monopole, conducting to a symmetric antenna configuration.

Both monopoles are fed by a coplanar waveguide through a transmission line, with a width of $2 \mathrm{~mm}$, length of $15 \mathrm{~mm}$ and a gap of $0.25 \mathrm{~mm}$. The antenna is designed to a $50 \mathrm{ohm}$ impedance, and details of optimized parameters of the proposed antenna are presented in Table I.

First of all, the radiating element is composed of a symmetrical folded monopole achieving prevalently the lower resonant frequency of $2.45 \mathrm{GHz}$. At this first resonant frequency, the monopole resonant path has a length of $64.9 \mathrm{~mm}$, which represents $0.53^{*}$ Lambda1. Later, a monopole was introduced to achieve successfully the high resonant frequency at $5.8 \mathrm{GHz}$. At this second resonant frequency, the resonant path of a monopole has a length of $7.5 \mathrm{~mm}$, representing $0.145^{*} \mathrm{Lambda} 2$. Combining the two monopoles shifted significantly the values of both resonant frequencies. Therefore, a parametric study was required to obtain the optimized antenna parameters. However to shortly discuss the designed antenna, only varying the values of the parameters W5 and L8 will be investigated in terms of plots in this paper.

The simulated reflection coefficient of the proposed antenna, by varying the parameter W5, is plotted in Fig. 2 (a). Applying various modifications on the value of the slot length W5, both resonant frequencies are shifted. It is noted that, a small increase of W5 parameter from 1 to $3 \mathrm{~mm}$, affects the resonance behavior of the designed antenna at the higher and lower resonant frequency. For example, in this study case, lower resonant frequency tends to decrease (from 3.11 to $2.23 \mathrm{GHz}$ ) while higher resonant frequency remains to oscillate around $5.82 \mathrm{GHz}$. However it is shown that, by choosing the adequate value around $2.2 \mathrm{~mm}$ for the parameter $\mathrm{W} 5$, it leads to the desired resonant frequencies of 2.45 and $5.8 \mathrm{GHz}$.

The simulated reflection coefficient of the proposed antenna, by varying the parameter L8, is plotted in Fig. 2 (b). Similarly, applying various modifications on the value of the slot length L8, both resonant frequencies are shifted. It is noted that, a small increase of L8 parameter from 5 to $7 \mathrm{~mm}$ with a step of $1 \mathrm{~mm}$, also affects the resonance behavior of the designed antenna at the higher and lower resonant

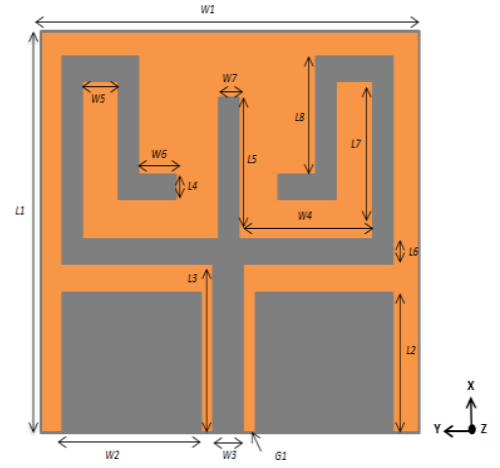

Fig. 1. Geometry of the planar dual-band double monopole antenna.

TABLE I

DETAILED ANTENNA PARAMETERS IN MM

\begin{tabular}{|c|c|c|c|}
\hline Parameter & Value & Parameter & Value \\
\hline L1 & 27.04 & W1 & 30 \\
\hline L2 & 14.5 & W2 & 10 \\
\hline L3 & 15.018 & W3 & 2 \\
\hline L4 & 1.07 & W4 & 9.395 \\
\hline L5 & 7.5 & W5 & 2.2 \\
\hline L6 & 1.07 & W6 & 3.27 \\
\hline L7 & 8.9 & W7 & 1.07 \\
\hline
\end{tabular}




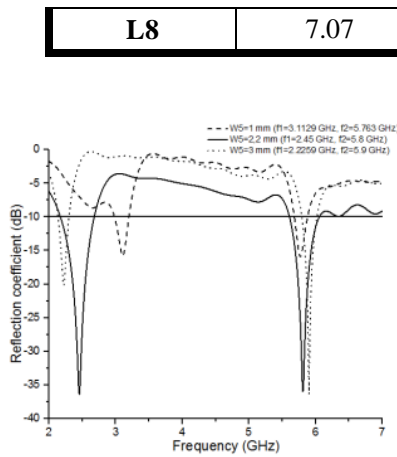

(a) (b)

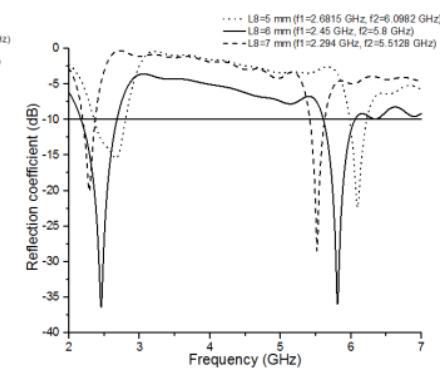

Fig. 2. Simulated reflection coefficient of the proposed dual-band double monopole antenna, by varying (a) W5 and (b) L8.

frequency. For example, the lower resonant frequency tends to decrease from 2.68 to $2.29 \mathrm{GHz}$, and likewise higher resonant frequency decreases from 6.1 to $5.51 \mathrm{GHz}$. However it is shown that, choosing the adequate value of $6 \mathrm{~mm}$ for the parameter L8, leads to the desired resonant frequencies of 2.45 and $5.8 \mathrm{GHz}$.

It is clearly seen that, varying effectively and properly the resonant paths: Length of the monopole 1 (Lm1), which is the symmetrical folded monopole and the length of the monopole 2 ( $\mathrm{Lm} 2)$, which is the other monopole (in terms of length, weight and shape), the resonant frequencies can achieve the accurate values of 2.45 and $5.8 \mathrm{GHz}$.

The length and width of all slots composing the symmetrical folded and other monopoles tune both the lower and higher resonant frequency of the proposed antenna. The input impedance is about $51.8-\mathrm{j} 5.8$ and $46.3+\mathrm{j} 3.6$ at the resonant frequencies 2.45 and $5.8 \mathrm{GHz}$, respectively. Impedance matching results show that the proposed antenna has a slightly capacitive and an inductive behavior at the resonant frequencies 2.45 and $5.8 \mathrm{GHz}$, respectively.

Moreover, the simulated surface current distributions of the proposed dual-band double monopole antenna at the 2.45 and $5.8 \mathrm{GHz}$ resonant frequencies are presented in Fig. 3, to provide an overall view of the current repartition over the proposed antenna. As discussed previously, it is clearly shown that symmetrical folded monopole is responsible of the first resonant frequency at $2.45 \mathrm{GHz}$, since current path shows a high current intensity. While at $5.8 \mathrm{GHz}$, current path shows different current directions in distant parts. For example, at the part A, currents are yielded in opposite directions. While at the parts B, currents came from opposite directions cancelling each other. Therefore the monopole is responsible of the excitation of the second resonant frequency $5.8 \mathrm{GHz}$. Furthermore, strong current distribution appears in the radiating element and also in the ground plan. Therefore, any change applied on the ground plane dimensions, including the gap parameter, will affect significantly the antenna impedance matching and consequently the antenna resonance behavior. The symmetric configuration allows the antenna to radiate symmetrically at different sides, providing an omnidirectional radiation pattern. The maximum 3D directivity of the planar antenna is $2.47 \mathrm{dBi}$ and $3.83 \mathrm{dBi}$ at 2.4 GHz and $5.8 \mathrm{GHz}$, respectively as presented in Fig. 4.

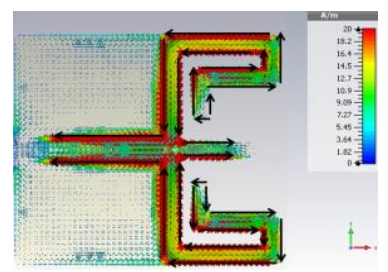

(a)

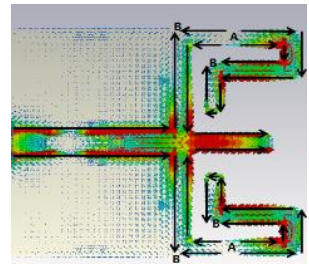

(b)
Fig. 3. Surface current distributions of the planar dual-band double monopole antenna at (a) 2.45 and (b) $5.8 \mathrm{GHz}$.

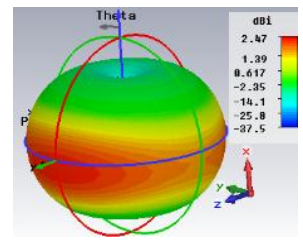

(a)

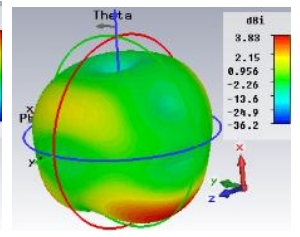

(b)
Fig. 4. Directivity of the planar dual-band double monopole antenna in free-space at $2.4 \mathrm{GHz}$ and $5.8 \mathrm{GHz}$.

\section{B. Measurement results}

To validate the working mechanism and the performances of the designed dual-band double monopole antenna, a prototype was made and measured as illustrated in Fig. 5. The measured and simulated reflection coefficients of the proposed antenna are plotted in Fig. 6. To measure impedance matching, the Vector Network Analyzer (VNA) Agilent PNA E8358A was used. The plots show that, the simulated $-10 \mathrm{~dB}$ impedance bandwidths are $310 \mathrm{MHz}(2.3-2.61 \mathrm{GHz})$ and $210 \mathrm{MHz}(5.71-5.92 \mathrm{GHz})$, representing $12.62 \%$ and $3.6 \%$ relative bandwidths at 2.45 and $5.8 \mathrm{GHz}$, respectively. While the measured bandwidths are $180 \mathrm{MHz}(2.43-2.61 \mathrm{GHz})$ and $260 \mathrm{MHz}(5.67-$ $5.93 \mathrm{GHz}$ ), representing $7.14 \%$ and $4.48 \%$ relative $-10 \mathrm{~dB}$ impedance bandwidths at 2.45 and $5.8 \mathrm{GHz}$, respectively. At the lower band, the measured reflection coefficient achieved $-20 \mathrm{~dB}$, while at the upper band $-23 \mathrm{~dB}$ is achieved. Similarly, these simulated values correspond to $27.5 \mathrm{~dB}$ and $-27 \mathrm{~dB}$ at 2.45 and $5.8 \mathrm{GHz}$, respectively.

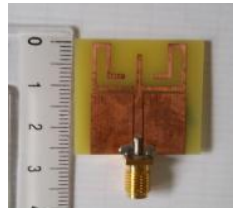

(a)

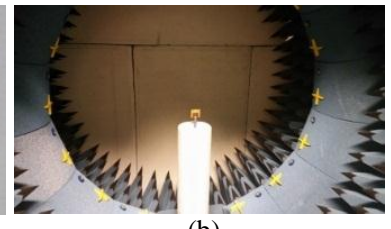

(b)
Fig. 5. Photograph of (a) the proposed planar antenna prototype with a scale and (b) the Satimo StarLab antenna measurement system.

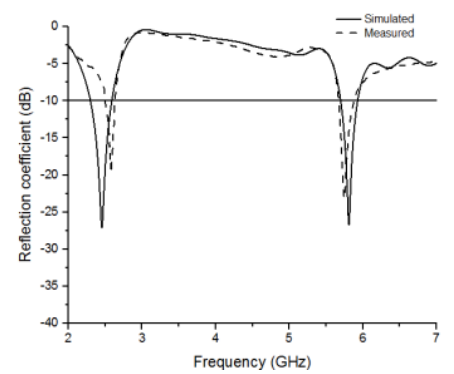

Fig. 6. Simulated and measured reflection coefficient of the planar dual-band double monopole antenna. 


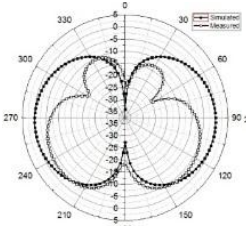

(a)

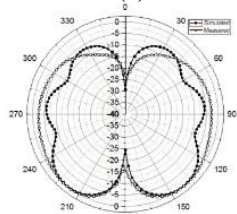

(d)

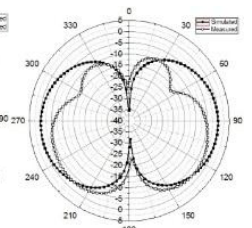

(b)

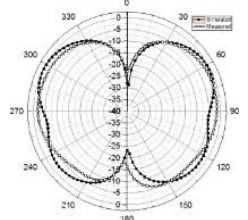

(e)

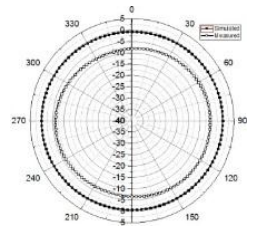

(c)

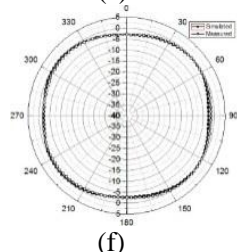

Fig. 7. Simulated and measured radiation patterns of the planar dual-band double monopole antenna in $\mathrm{dB}$ in free space at 2.45 (a) $\mathrm{Phi}=0^{\circ}$ (b) $\mathrm{Phi}=90^{\circ}$ (c) Theta $=90^{\circ}$ and at $5.8 \mathrm{GHz}$ (d) $\mathrm{Phi}=0^{\circ}$ (e) Phi $=90^{\circ}$ (f) Theta $=90^{\circ}$.

Measurements reveal that a slight frequency shift appears at the lower resonant frequency from 2.45 to $2.58 \mathrm{GHz}$. However, the high resonant frequency remains stable. In other words, the measured bandwidth was decaled slightly up at the lower resonant frequency, while at the higher resonant frequency, the bandwidth remains the same. According to the presented results, simulated and measured results are in quite agreement. The resulted narrow band effect shown at both lower and upper bands allows the antenna to avoid any interference with the other frequency bands.

The radiation pattern was measured by using Satimo Starlab. The simulated and measured radiation patterns of the proposed dual-band double monopole antenna are illustrated in Fig. 7. Different graphs are presented for different cuts, according to $\mathrm{Phi}=0^{\circ}, \mathrm{Phi}=90^{\circ}$ and Theta $=90^{\circ}$ cuts, at the resonant frequencies 2.45 and $5.8 \mathrm{GHz}$. As it can be observed, both simulated and measured results are symmetric due to the symmetric antenna configuration. The measured results demonstrate that the proposed dual-band double monopole antenna has an omnidirectional radiation pattern at the lower and higher resonant frequencies. The simulated maximum gains of the proposed dual-band double monopole antenna are $2.52 \mathrm{dBi}$ and $3.84 \mathrm{dBi}$ at 2.45 and $5.8 \mathrm{GHz}$, respectively. The inaccuracy tolerance of the fabrication process and the errors caused by the measurement process might be among the main reasons behind the difference (regarding the reflection coefficient and the radiation pattern plots) between simulated and measured results. Another factor can be responsible of the slight shift, is the effect of the coaxial cable in the measurement setup, which can be clearly seen in the measured radiation patterns.

\section{REFLECTOR-BACKED CONFIGURATION}

In this section, a metallic plate was placed at the back of the planar antenna serving as reflector, as illustrated in Fig. 8. The reflector is preferred to minimize the side lobes of the antenna radiation and hence reduce any possible interference with other technologies using the same ISM band in practical scenario. Additionally, this new antenna configuration helps to foster the antenna directivity towards Z-direction which is very important for on-body applications. In this sense, the size of the reflector and the antenna

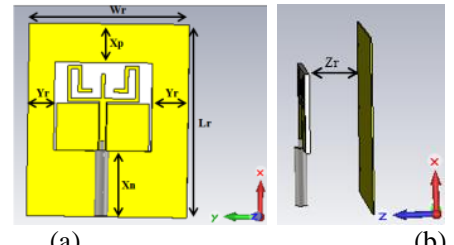

(a)

(b)

Fig. 8. Geometry of the reflector-backed dual-band double monopole antenna. (a) Front view (b) Side view.

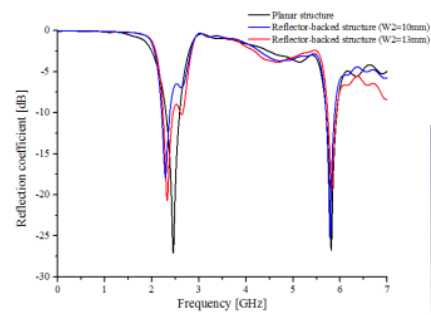

(a)

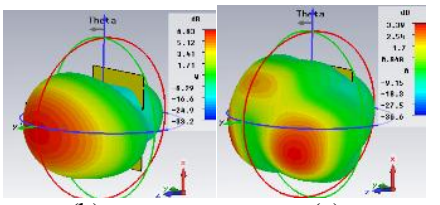

(b) (c)
Fig. 9. (a) Simulated reflection coefficient comparison of the planar and reflector-backed dual-band double monopole antenna in free-space. (b) Directivity of the reflector-backed dual-band double monopole antenna in free-space at (b) $2.4 \mathrm{GHz}$ and (c) $5.8 \mathrm{GHz}$ in free-space.

position are relevant criterions to pay attention. After a sensitive tuning parameter study based on the parameters $\mathrm{Wr}, \mathrm{Lr}, \mathrm{Yr}, \mathrm{Xp}, \mathrm{Xn}$ and $\mathrm{Zr}$, the optimized values are $50 \mathrm{~mm}$, $60 \mathrm{~mm}, 20 \mathrm{~mm}, 13 \mathrm{~mm}, 20 \mathrm{~mm}$ and $20 \mathrm{~mm}$ respectively. For brevity, the detailed parameter analysis is not present in this paper.

From Fig. 9 (a), it seems that the cavity did not alter that much the two covered ISM bands, especially the upper band of $5.72-5.93 \mathrm{GHz}$ (determined by $-10 \mathrm{~dB}$ ). A rough frequency shift it remarked at the lower frequency band, particularly by increasing the parameter $\mathrm{W} 2$ from $10 \mathrm{~mm}$ to $13 \mathrm{~mm}$ which resulted in a little frequency increase from $2.38 \mathrm{GHz}$ to $2.50 \mathrm{GHz}$. The aim of the proposed antenna to cover the full $2.4 \mathrm{GHz}$ ISM band, therefore the parameter W2 was set to $13 \mathrm{~mm}$ which lead to a covered bandwidth of 2.23-2.50 GHz and 5.72-5.93 GHz. The simulated 3D realized gain of the reflector antenna configuration at $2.4 \mathrm{GHz}$ and $5.8 \mathrm{GHz}$ is depicted in Fig. 9 (b) and (c), respectively. The $3 \mathrm{D}$ radiation pattern shows clearly the good antenna directivity of $6.83 \mathrm{~dB}$ and $3.39 \mathrm{~dB}$ towards Zdirection at $2.4 \mathrm{GHz}$ and $5.8 \mathrm{GHz}$, respectively. These findings promote the efficient use of the proposed structure for receiving on-body application.

\section{ON-BODY AND IN-BODY SIMULATIONS}

In this section, the reflector-backed antenna was situated at the closest distance, assumed $d s a=4 \mathrm{~mm}$, from a multilayer model, as illustrated in Fig. 10, and results were compared to voxel model using Laura CST model 2018. The boundary conditions are accurately set to avoid any over power flow at the extreme borders of the multi-layer and voxel models. Upper frequencies are well known in literature to yield high path loss and consequently significant signal attenuation. Therefore, in this on-body investigation, the analyses using $2.4 \mathrm{GHz}$ lower frequency are compared to the ones using $5.8 \mathrm{GHz}$. The human body effect is emulated using several body tissues with dielectric properties corresponding to $2.4 \mathrm{GHz}$ and $5.8 \mathrm{GHz}$ [14-17], as listed in Table II. The combined human tissues are "Skin", "Fat1", "Muscle", "Fat2", "Small Intestine Wall" and "Small Intestine", appearing in this order from the antenna side, as described in Fig. 10 (b). The tissue thicknesses are provided in Table II, for "Fat2 tissue" two cases are distinguished and 
named P1 and P2 for respective thicknesses of $27 \mathrm{~mm}$ and $73 \mathrm{~mm}$.

By situating the reflector-backed antenna very close to the multi-layer model, the upper band was widened covering 5.70-6.17 GHz for both person cases $\mathrm{P} 1$ and $\mathrm{P} 2$ as presented in Fig. 11 (a). However, at the lower band, the covered $10 \mathrm{~dB}$ bandwidth is almost the same around $2.23-2.50 \mathrm{GHz}$, regardless the study case ( $\mathrm{P} 1$ and $\mathrm{P} 2)$. This proves that the change of the tissue thickness does not affect the antenna performances in terms of return loss. This analysis is completed with the input impedance results of the antenna next to the human model. The input impedance results at $2.4 \mathrm{GHz}$ and $5.8 \mathrm{GHz}$ are $33.27-\mathrm{j} 5.67 \mathrm{ohm}$ and $57+\mathrm{j} 15.68$ $\mathrm{ohm}$, respectively as presented in Fig. 11 (b). These results show that the antenna has a capacitive behavior at $2.4 \mathrm{GHz}$ and inductive one at $5.8 \mathrm{GHz}$ for both study cases (P1 and $\mathrm{P} 2)$. Besides, one can note that the antenna resistance has decreased to $33.27 \mathrm{ohm}$ at $2.4 \mathrm{GHz}$ and increased to $57 \mathrm{ohm}$ at $5.8 \mathrm{GHz}$, compared to the free-space resistance of $56 \mathrm{ohm}$. This difference can be related to the dielectric properties of the human layers which are emulated at $2.4 \mathrm{GHz}$ and $5.8 \mathrm{GHz}$. Close results are shown using voxel model. The 3D directivity is illustrated in Fig. 12, showing maximum values of $7.95 \mathrm{~dB}, 7.82 \mathrm{~dB}$ at $2.4 \mathrm{GHz}$ and $7.51 \mathrm{~dB}, 7.12 \mathrm{~dB}$ at $5.8 \mathrm{GHz}$ using multi-layer and voxel model, respectively. These finding was followed by the evaluation of the antenna radiation penetration through the considered human model as described in Fig. 13. The study relays on placing several power probes at different tissue interfaces (presenting the change of the dielectric properties), appearing as red circles in Fig. 13 (a). The power values were calculated for both cases $\mathrm{P} 1$ and $\mathrm{P} 2$ at the frequencies of interest $2.4 \mathrm{GHz}$, $2.45 \mathrm{GHz}$ and $5.8 \mathrm{GHz}$ and grouped in Table III. By comparing the total power consumed from the start of the skin layer to the end interface of SI, it is apparent the average power is between $41 \mathrm{~dB}$ and $54 \mathrm{~dB}$ at $2.4 \mathrm{GHz}$ and $2.45 \mathrm{GHz}$, respectively. This value is found between 64 and $89 \mathrm{~dB}$ at $5.8 \mathrm{GHz}$ for P1 and P2 cases, respectively. This power consumption value is distributed at the interfaces of skin, Fat1, Muscle, Fat2, Small intestine Wall, Start of the small intestine, middle of the small intestine and end of the small intestine tissues. Back to theory, one would expect a power field increase with the frequency increase which is verified here.

Later, the amount of power consumed by the planar multi-layer model P1 and P2 is estimated using the transmission parameter (S12) by placing two proposed antennas at the extreme interfaces of the model (at the closest distance from Skin and SI layers), as illustrated in Fig. 13 (b). Reported transmission parameter values are collected in Table IV. The table shows that the path loss is increased with the frequency increase from $2.4 \mathrm{GHz}$ to $5.8 \mathrm{GHz}$ for P1 and P2 human models. Furthermore, the path loss is assumed in $39-48 \mathrm{~dB}$ range at $2.4 / 2.45 \mathrm{GHz}$ against $60-80 \mathrm{~dB}$ range at $5.8 \mathrm{GHz}$. This time, the results are more predictable and can be considered reliable for both frequencies $2.4 / 2.45 \mathrm{GHz}$ and $5.8 \mathrm{GHz}$. Even more, by comparing the total power-field [Skin-SI] with the transmission coefficient values from Table III and Table IV, respectively one can clearly remark/notice the close convergence of both values at $2.4 / 2.45 \mathrm{GHz}$ and $5.8 \mathrm{GHz}$. This alone confirms that the $6.83 \mathrm{~dB}$ gain at $2.4 / 2.45 \mathrm{GHz}$ and $3.39 \mathrm{~dB}$ gain at $5.8 \mathrm{GHz}$ are good and sufficient to inform accurately on the power consumption by the human tissues. When evaluating the power consumed using voxel model, only $15 \mathrm{~dB}$ and $31 \mathrm{~dB}$ averaged power is reported in Table III from skin to the end of SI organ. Furthermore, the repartition of this power is calculated at the different locations mentioned as blue circles in Fig. 13 (c). Once again, it is clearly seen the power increase with the frequency increase using voxel model. However, in stark contrast to the multi-layer results, the averaged power is lower and more logical as the signal will propagate via easier path. Therefore, it is recommendable and desirable to evaluate this issue using other voxel models in the following investigations in the future. Besides, the proposed dual-band antenna does not present any risk on the person holding it for measurement, this is supported by the calculated maximum SAR (10g) values of $0.066 \mathrm{~W} / \mathrm{kg}$ and $0.098 \mathrm{~W} / \mathrm{kg}$ at $2.4 \mathrm{GHz}$ and $5.8 \mathrm{GHz}$, respectively. SAR values were calculated in compliance with IEEE C95.3 standard provided by CST MW software with a chosen input power of $0.00316 \mathrm{~W}$. It is clearly seen that the SAR increases with the frequency increase from $2.4 \mathrm{GHz}$ to $5.8 \mathrm{GHz}$.

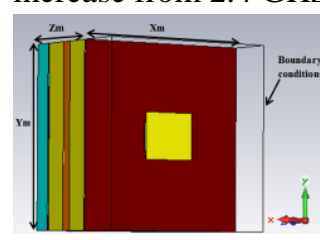

(a)

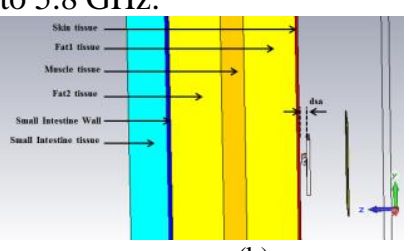

(b)
Fig. 10. The antenna emplacement for on-body simulations. (a) Perspective view (b) Side view.

TABLE II

DIELECTRIC PROPERTIES AT 2.4 / 5.8 GHZ AND THICKNESSES OF THE HUMAN TISSUES

\begin{tabular}{|c|c|c|c|c|}
\hline Tissues & Permittivity & $\begin{array}{c}\text { Conductivity } \\
(\mathrm{S} / \mathrm{m})\end{array}$ & $\begin{array}{c}\text { Density } \\
(\mathrm{kg} / \mathrm{m} 3)\end{array}$ & $\begin{array}{c}\text { Thickness } \\
(\mathrm{mm})\end{array}$ \\
\hline Skin & $38.1 / 35.1$ & $1.44 / 3.72$ & 1109 & 1.4 \\
\hline Fat1 & $10.8 / 9.86$ & $0.261 / 0.83$ & 911 & 27 \\
\hline Muscle & $52.8 / 48.5$ & $1.71 / 4.96$ & 1090 & 12 \\
\hline Fat2 & $10.8 / 9.86$ & $0.261 / 0.83$ & 911 & $27 / 73$ \\
\hline $\begin{array}{c}\text { Small } \\
\text { Intestine } \\
\text { Wall }\end{array}$ & $52.8 / 48.5$ & $1.71 / 4.96$ & 1045 & 2 \\
\hline $\begin{array}{c}\text { Small } \\
\text { Intestine }\end{array}$ & $54.4 / 48.7$ & $3.13 / 6.75$ & 1030 & 20 \\
\hline
\end{tabular}

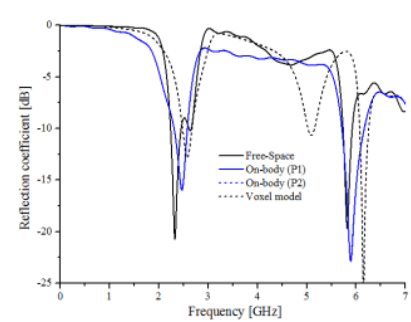

(a)

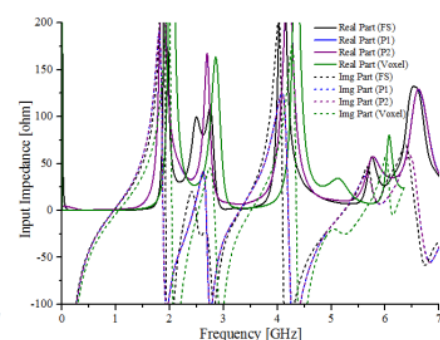

(b)
Fig. 11. Simulated (a) reflection coefficient and (b) input impedance comparison of reflector-backed dual-band double monopole antenna next to $\mathrm{P} 1 / \mathrm{P} 2$ and voxel model.

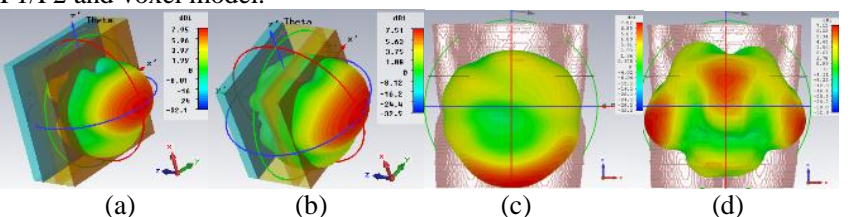

Fig. 12. Directivity of the reflector-backed dual-band double monopole antenna at (a) $2.4 \mathrm{GHz}$ and (b) $5.8 \mathrm{GHz}$ next to the planar multi-layer model and at (c) $2.4 \mathrm{GHz}$ and (d) $5.8 \mathrm{GHz}$ next to voxel model. 


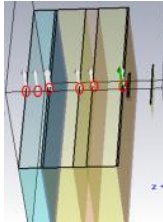

(a)

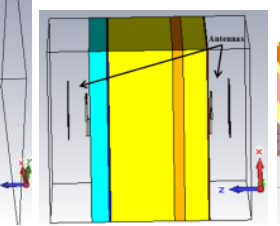

(b)

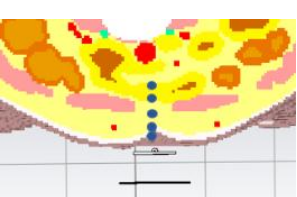

(c)
Fig. 13. (a) Power probes emplacement inside the multi-layer model. (b) Power consumption of the multi-layer model using two proposed antennas for P1 and P2. (c) Power probes emplacement inside the voxel model.

TABLE III: POWER-FIELD USING PLANAR MULTI-LAYER AND VOXEL MODELS AT DIFFERENT FREQUENCIES

\begin{tabular}{|c|c|c|c|}
\cline { 2 - 4 } \multicolumn{1}{c|}{$\begin{array}{c}\text { Power-Field } \\
\text { [dB] }\end{array}$} & $2.4 \mathrm{GHz}$ & $2.45 \mathrm{GHz}$ & $5.8 \mathrm{GHz}$ \\
\hline Skin Surface & $28.61 / 28.62$ & $28.02 / 28.01$ & $10.74 / 10.75$ \\
\hline Fat1 Surface & $24.79 / 24.83$ & $24.29 / 24.26$ & $6.1 / 6.1$ \\
\hline Muscle Surface & $17.53 / 17.56$ & $17.26 / 17.26$ & $3.50 / 3.50$ \\
\hline Fat2 Surface & $11.30 / 11.65$ & $11.02 / 11.24$ & $-11.87 /-11.90$ \\
\hline SI Wall & $3.64 /-7.39$ & $3.23 /-7.11$ & $-26.94 /-52.80$ \\
\hline SI Start & $2.75 /-8.08$ & $2.51 /-8.33$ & $-29.51 /-57.93$ \\
\hline SI Middle & $-4.75 /-16.95$ & $-5.01 /-17.29$ & $-47.89 /-73.36$ \\
\hline SI End & $-12.45 /-26.33$ & $-12.87 /-26.51$ & $-53.49 /-78.95$ \\
\hline Total [Skin-SI] & $\mathbf{4 1 . 0 6 / 5 4 . 9 6}$ & $\mathbf{4 0 . 8 9 / 5 4 . 5 2}$ & $\mathbf{6 4 . 2 3} / \mathbf{8 9 . 7}$ \\
\hline Power-Field & & Laura voxel model & \\
\hline [dB] & $2.4 \mathrm{GHz}$ & $2.45 \mathrm{GHz}$ & $5.8 \mathrm{GHz}$ \\
\hline Just before Skin & -45.4 & -45.6 & -50.1 \\
\hline Skin tissue & -46.9 & -47.2 & -52.5 \\
\hline Fat tissue & -47.1 & -47.9 & -54.5 \\
\hline Start of SI & -50.9 & -51.2 & -57.3 \\
\hline End of SI & -60.6 & -61.3 & -81.2 \\
\hline Total [Skin-SI] & $\mathbf{1 5 . 2}$ & $\mathbf{1 5 . 7}$ & $\mathbf{3 1 . 1}$ \\
\hline
\end{tabular}

TABLE IV: TRANSMISSION PARAMETER OF THE PROPOSED DUAL-BAND ANTENNA THROUGH THE MULTI-LAYER MODEL

\begin{tabular}{|l|l|l|l|}
\hline $\mathrm{S} 12[\mathrm{~dB}]$ & $2.4 \mathrm{GHz}$ & $2.45 \mathrm{GHz}$ & $5.8 \mathrm{GHz}$ \\
\hline $\mathrm{P} 1$ (On-body) & -39.33 & -39.77 & -60.67 \\
\hline P2 (On-body) & -47.77 & -48.15 & -81.34 \\
\hline
\end{tabular}

\section{CONCLUSION}

In this paper, a novel dual-band CPW-fed double monopole antenna is presented. Dual-band and omnidirectional radiation patterns are obtained at 2.45 and $5.8 \mathrm{GHz}$ using planar antenna structure. The lower resonant frequency of $2.45 \mathrm{GHz}$ is achieved basically by using a symmetrical folded monopole, whereas the upper resonant frequency at $5.8 \mathrm{GHz}$ is obtained by a short monopole. A prototype of the planar antenna configuration was fabricated and tested as first prototype trial. Later, the antenna performances were improved by introducing a reflector to the planar structure, which enhanced significantly the gain to $6.83 \mathrm{~dB}$ and $3.39 \mathrm{~dB}$ at $2.4 \mathrm{GHz}$ and $5.8 \mathrm{GHz}$, respectively. Besides the resulting reflector-backed antenna is directive at both $2.4 \mathrm{GHz}$ and $5.8 \mathrm{GHz}$ which is preferred to establish good communication with the capsule that is assumed to be travelling the small-intestine tract. These free-space investigations were followed by on-body studies using multi-layer and voxel models which validated the suitability of the proposed reflector-backed antenna to wireless capsule endoscopy localization systems. The presented results demonstrate that this double monopole antenna could be considered for 2.45 and $5.8 \mathrm{GHz}$ applications, since the antenna operates in the 2.4/5.8 ISM bands for medical Body Area Networks. These results will be validated by measurements as next step.

\section{ACKNOWLEDGMENT}

The findings of this paper are the result of the cooperation with Centre of Wireless Communications (CWC), Oulu University, Oulu, Finland. The work was financially supported by ERASMUS+ Project.

\section{REFERENCES}

[1] P. J. Soh, F. N. Giman, M. F. Jamlos, H. Lago, and A. A. Al-Hadi, "A C-slotted dual band textile antenna for WBAN applications," URSI Asia-Pacific Radio Science Conference (URSI AP-RASC), pp. $1621-1624,2016$

[2] X. -Q. Zhu, Y. -X. Guo, and W. Wu, "A compact dual band antenna for wireless Body-Area Network applications," IEEE Antennas and Wireless Propagation Letters, vol. 15, pp. 98 - 101, 2016

[3] W. Lee, and J. Choi, "A dual-band printed antenna with metal backcover for WBAN applications," IEEE International Symposium on Antennas and Propagation \& USNC/URSI National Radio Science Meeting, pp. 936 - 937, 2015

[4] Y. Hong, J. Tak, and J. Choi, "Dual-band dual-mode patch antenna for on-on-off WBAN applications," Electronics Letters, vol. 50, pp. $1895-1896,2014$

[5] A. T. Mobashsher, and R. W. Aldhaheri, "An improved uni-planar front-directional antenna for dual-band RFID readers," IEEE Antennas and Wireless Propagation Letters, vol. 11, pp. $1438-$ 1441, 2012.

[6] C. -C. Lin, E. -Z. Yu, and C. -Y. Huang, "Dual-band rhombus slot antenna fed by cpw for WLAN applications," IEEE Antennas and Wireless Propagation Letters, vol. 11, pp. 362 - 364, 2012

[7] S. Ahdi Rezaeieh, A. Abbosh, M. A. Antoniades, and S. Mustafa, "Dual-Band Dual-Polarized Antenna for Medical Applications", International Conference on Electromagnetics in Advanced Applications(ICEAA), pp. 1277 - 1279, 2013

[8] X. -Q. Zhu; Y. -X. Guo; and W. Wu, "A Novel Dual-band Antenna for Wireless Communication Applications", IEEE Antennas and Wireless Propagation Letters, Vol. 15, pp. 516-519, 2016.

[9] R. Li ; Y-. X. Guo and G. Du, "A Conformal Circularly Polarized Antenna for Wireless Capsule Endoscope Systems", IEEE Transactions on Antennas and Propagation, Vol. 66, I. 4, 2018.

[10] C. Liu ; Y-. X. Guo ; R. Jegadeesan ; and S. Xiao, "In Vivo Testing of Circularly Polarized Implantable Antennas in Rats", Vol. 14, 2015.

[11] Z. Bao ; Y-. X. Guo ; and R. Mittra, " Conformal Capsule Antenna with Reconfigurable Radiation Pattern for Robust Communications", IEEE Transactions on Antennas and Propagation, Vol. 66, I. 7, 2018.

[12] Y. Li ; Y-. Xin Guo ; and S. Xiao, "Orientation Insensitive Antenna with Polarization Diversity for Wireless Capsule Endoscope System", IEEE Transactions on Antennas and Propagation, Vol. 65, I. 7, 2017.

[13] Z. Bao ; Y-. X. Guo ; and R. Mittra, "An Ultrawideband Conformal Capsule Antenna With Stable Impedance Matching", IEEE Transactions on Antennas and Propagation, Vol. 65, I. 10, 2017.

[14] http://www.fcc.gov/oet/rfsafety/dielectric.html

[15] https://www.itis.ethz.ch/virtual-population/tissueproperties/ database/dielectric-properties/

[16] S.M. Bunce, A.P. Moore, A.D. Hough, "M-mode ultrasound: a reliable measure of trans-versus abdominis thickness?", Clinical Biomechanics, vol. 17, pp. 315-317, 2002.

[17] O. Akkus, A. Oguz, M. Uzunlulu and M. Kizilgul, "Evaluation of Skin and Subcutaneous Adipose Tissue Thickness for Optimal Insulin Injection", Diabetes \& Metabolism, pp. 3-8, 2012. 